\title{
The neural correlates of fatigue: an exploratory imaginal fatigue provocation study in chronic fatigue syndrome
}

\author{
X. Caseras ${ }^{1,2 *}$, D. Mataix-Cols ${ }^{2,3}$, K. A. Rimes ${ }^{2}$, V. Giampietro ${ }^{4}$, M. Brammer ${ }^{4}$, F. Zelaya ${ }^{4}$, T. Chalder ${ }^{2}$ \\ and E. Godfrey ${ }^{3}$ \\ ${ }^{1}$ Unitat de Psicologia Mèdica, Institut de Neurociències, Universitat Autònoma de Barcelona, Catalonia, Spain \\ ${ }^{2}$ Division of Psychological Medicine and Psychiatry, King's College London, Institute of Psychiatry, London, UK \\ ${ }^{3}$ Department of Psychology, King's College London, Institute of Psychiatry, London, UK \\ ${ }^{4}$ Centre for Neuroimaging Sciences, King's College London, Institute of Psychiatry, London, UK
}

Background. Fatigue is the central symptom in chronic fatigue syndrome (CFS) and yet very little is known about its neural correlates. The aim of this study was to explore the functional brain response, using functional magnetic resonance imaging (fMRI), to the imaginal experience of fatigue in CFS patients and controls.

Method. We compared the blood oxygen level dependent (BOLD) responses of 12 CFS patients and 11 healthy controls to a novel fatigue provocation procedure designed to mimic real-life situations. A non-fatiguing anxiety-provoking condition was also included to control for the non-specific effects of negative affect.

Results. During the provocation of fatigue, CFS patients reported feelings of both fatigue and anxiety and, compared to controls, they showed increased activation in the occipito-parietal cortex, posterior cingulate gyrus and parahippocampal gyrus, and decreased activation in dorsolateral and dorsomedial prefrontal cortices. The reverse pattern of findings was observed during the anxiety-provoking scenarios.

Conclusions. The results may suggest that, in CFS patients, the provocation of fatigue is associated with exaggerated emotional responses that patients may have difficulty suppressing. These findings are discussed in relation to the cognitive-behavioural model of CFS.

Received 30 May 2007; Revised 17 March 2008; Accepted 31 March 2008; First published online 30 April 2008

Key words: Anxiety, chronic fatigue syndrome, fatigue, fMRI.

\section{Introduction}

Chronic fatigue syndrome (CFS) is a complex and medically unexplained illness that is characterized by severe and prolonged disabling fatigue and a combination of symptoms, including sleep disturbance, musculoskeletal pain and impairment in concentration and short-term memory (Fukuda et al. 1994). Fatigue is the central symptom of CFS and yet very little research has been devoted to it. Previous research in CFS emphasizes the role of the central nervous system in the subjective perception of fatigue (Kent-Braun et al. 1993; Georgiades et al. 2003). This is not surprising considering the effect that factors such as emotion, attention and motivation can have on fatigue perception (St Clair Gibson et al. 2003).

\footnotetext{
* Address for correspondence: Dr X. Caseras, PO 69, Section of Cognitive Neuropsychiatry, Institute of Psychiatry, De Crespigny Park, London SE5 8AF, UK.

(Email: xavier.caseras@iop.kcl.ac.uk)
}

Like chronic pain sufferers, CFS patients have been shown to avoid physical activities (van der Werf et al. 2000) and to score highly on the Tampa Scale of Kinesiophobia, or phobia of physical movement and activity (Silver et al. 2002). CFS patients believe that physical activity will increase their fatigue, leading to avoidance in a similar way that phobia-related cognitions lead to avoidance in phobic patients (Deale et al. 1998; Chalder et al. 2000). It has also been shown that exercise lowers the pain threshold in CFS patients (Whiteside et al. 2004), probably making any physical sensation a painful experience. If this is the case, it is not difficult to understand that for most CFS patients physical activity can be a highly demanding and emotional situation, leading to fear and avoidance. In fact, the cognitive-behavioural model of CFS highlights the importance of fear and avoidance in the aetiology and/or maintenance of the disorder (Chalder et al. 2000) and cognitive-behavioural therapy (CBT) techniques aimed at tackling these 
symptoms are effective in reducing fatigue (Whiting et al. 2001). Moreover, anxiety is a major co-morbidity in CFS (e.g. Fischler et al. 1997) and a high proportion of patients with anxiety disorders complain of fatigue (e.g. Angst \& Dobler-Mikola, 1985), reinforcing the link between CFS and anxiety disorders.

Little is known about the neural correlates of fatigue. Recent neuroimaging studies have compared brain responses to cognitively challenging tasks between CFS patients and healthy controls (Lange et al. 2005; Caseras et al. 2006; Cook et al. 2007) but only one of these studies correlated subjective fatigue scores with brain activity during a working memory task (Cook et al. 2007). To our knowledge, no previous studies have used in vivo functional neuroimaging techniques to study brain activation during the experimental provocation of imagined physical fatigue in CFS. Symptom provocation is a useful research paradigm because it allows the testing of important questions that cannot be answered if the patients are asymptomatic. It has proven invaluable in neuroimaging studies of a variety of problems such as obsessive-compulsive disorder (Mataix-Cols et al. 2004) or depression (Beauregard et al. 2006). The current functional magnetic resonance imaging (fMRI) study used a novel fatigue provocation procedure to examine the neurophysiological basis of the experience of imaginal fatigue in healthy volunteers and in CFS patients. Because fatigue is often accompanied by negative emotional states, we also included an anxiety-provoking condition to control for any nonspecific emotional effects on brain activation. We predicted that, compared to healthy controls, CFS patients would show enhanced neural responses in brain regions implicated in the representation of pain and internal somatic states (i.e. insular cortex, anterior cingulate gyrus, somatosensory cortex, posterior parietal cortex). In addition, we expected higher activations in limbic and paralimbic regions in CFS patients compared with controls, consistent with the accompanying negative affect during the provocation of fatigue.

\section{Method}

\section{Participants}

Thirteen right-handed CFS patients (eight women; age range 22-45 years) and 12 right-handed healthy controls (eight women; age range 24-45 years) were included in the study. The CFS patients were recruited between February and October 2005 from the CFS Research and Treatment Units of King's College London $(n=12)$ and St Bartholomew's Hospital $(n=1)$, and fulfilled strict Centers for Disease Control and
Prevention (CDC) criteria for CFS (Fukuda et al. 1994). The patients were referred by their general practitioner (GP) to the CFS Units with a suspected diagnosis of CFS. At the CFS Units a physician conducted a complete clinical interview and reviewed the results of the required tests to exclude other conditions, before accepting the diagnosis of CFS as valid. Finally, a clinical psychologist independently confirmed the diagnosis using a checklist of the CDC criteria.

Control participants were recruited by advertisement and included staff at the Institute of Psychiatry and members of the wider local community. Exclusion criteria were present or past history of CFS (controls only) or any psychiatric disorder, history of brain injury, claustrophobia, suspected pregnancy, presence of any metallic implants and regular physical exercise. We selected controls with a low to medium level of physical fitness (i.e. those reporting practising sports 'almost never' or 'once a month or less') to make them as comparable as possible to the CFS group.

Two participants (one control and one patient) reported not being able to successfully complete the imagination task and were therefore excluded from the analysis. The final sample included 12 patients and 11 controls.

The study was approved by the ethics committees of the South London and Maudsley National Health Service (NHS) Trust and St Bartholomew's Hospital. All individuals gave written informed consent to participate.

\section{Measures}

After confirming the presence of CFS, an experienced clinician administered the Mini International Neuropsychiatric Interview (MINI; Sheehan et al. 1998) to rule out the presence of major psychiatric co-morbidity. All participants completed the Chalder Fatigue Scale (ChFS; Chalder et al. 1993), the Physical Functioning Scale from the Short Form Health Survey SF-36 (Ware \& Sherbourne, 1992), the Work and Social Adjustment Scale (WSAS; Mundt et al. 2002; Mataix-Cols et al. 2005) and the Hospital Anxiety and Depression Scale (HADS; Zigmond \& Snaith, 1983).

\section{Procedure}

Fatigue and anxiety provocation task

Subjects were requested to imagine themselves in different situations while viewing video-clips previously selected to induce fatigue, anxiety or relaxation (see Fig. 1). To enhance the participants' experience, the video-clips were filmed from a first person's point of view and were back-projected onto 


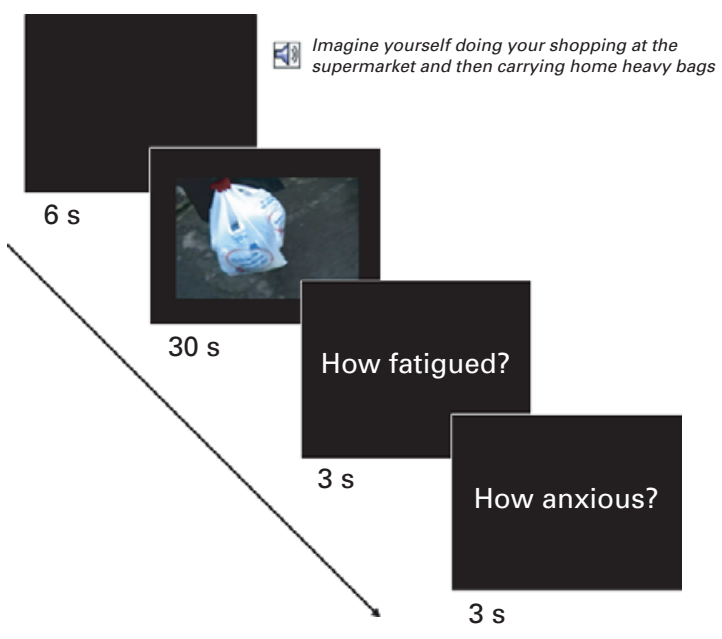

Fig. 1. Design of the study.

a screen that could be seen through a mirror mounted on the head-coil.

Before each video-clip, participants heard a prerecorded voice file through high-fidelity pneumatic headphones telling them which situation they were required to imagine. For example, the instruction 'imagine yourself doing your shopping at the supermarket and then carrying home heavy bags' preceded a videoclip showing a person carrying heavy shopping bags. Similarly, for the anxiety-provoking videos, the instruction 'imagine yourself standing on the edge of a high cliff and looking over the edge' was followed by a video-clip showing the feet of a person on the edge of a cliff. Finally, for the relaxation videos (the control condition), the instruction 'imagine yourself sitting in a comfy armchair drinking a nice cup of tea' was followed by a clip of somebody holding a cup of tea with her feet up. After each video, the participants were requested to produce two separate subjective ratings of fatigue (on a visual analogue scale of $0-10$, where $0=$ no fatigue and $10=$ extreme fatigue) and anxiety (on a visual analogue scale of $0-10$, where $0=$ no anxiety and $10=$ extreme anxiety) after imagining themselves in each situation (Fig. 1). All participants received a training session prior to scanning to ensure that they understood the task.

A pilot study involving seven CFS patients and four healthy controls (not included in this study) led to the selection of the final four fatigue-inducing, four anxiety-inducing and eight relaxing videos among an originally larger pool of video-clips. Fatigue and Anxiety videos were $30 \mathrm{~s}$ long and Relax videos $16 \mathrm{~s}$ long. The videos were separated by $12 \mathrm{~s}: 6 \mathrm{~s}$ of a black screen preceded each video during which time the pre-recorded voice was played, and $6 \mathrm{~s}$ following each video during which time the subjects were requested to rate their fatigue and anxiety ( $3 \mathrm{~s}$ per rating). The relaxation videos were always interspersed between the fatigue and anxiety videos to prevent any carryover effects. Two different order playlists were created and used randomly: Relax/Fatigue/Relax/Anxiety (four times) and Relax/Anxiety/Relax/Fatigue (four times). The specific video-clip to be played on each occasion was chosen randomly. A random half of the participants saw one playlist, while the other half saw the other.

\section{Image acquisition}

Single-shot, gradient echo, echo-planar imaging was used to acquire $184 \mathrm{~T} 2^{*}$-weighted image volumes on a $1.5 \mathrm{~T}$ GE Excite MRI scanner (General Electric, Milwaukee, WI, USA) at the Centre for Neuroimaging Sciences of the Institute of Psychiatry, King's College London. For each volume, 25 non-contiguous axial planes parallel to the inter-commissural plane were collected with the following parameters: $\mathrm{TR}=$ $2000 \mathrm{~ms}$, TE $=40 \mathrm{~ms}$, slice thickness $=5 \mathrm{~mm}$, slice skip $=0.5 \mathrm{~mm}$, field of view $(F O V)=24 \mathrm{~cm}$, image acquisition matrix $64 \times 64$, yielding an in-plane spatial resolution of $3.75 \mathrm{~mm}$. Four dummy acquisitions were also made at the beginning of each run to set the longitudinal magnetization into steady state. The start of the task was triggered by the scanner immediately at the end of the dummy acquisition.

Fifteen whole-brain volumes were acquired during the fatigue- and anxiety-inducing videos, and eight volumes during the relaxing videos. During the $6 \mathrm{~s}$ of verbal instructions preceding each video, three 'dummy-volumes' were excited using the same radiofrequency envelope and gradient slice selection parameter, with the same repetition time of $2 \mathrm{~s}$ to allow magnetization to reach an equilibrium amplitude prior to the next period of data acquisition. The frequency-encoding gradient was turned off during this period to minimize acoustic noise and ensure that the instructions were heard clearly by the subjects. After each video a 6-s period of silence was introduced to help the experimenter to hear the ratings of fatigue and anxiety reported by the participants. The three dummy-volumes before and after each video were modelled in the analyses and treated as a nuisance variable.

To facilitate co-registration of the fMRI data in standard space, we also acquired during each session a 43-slice, high-resolution echo planar image of the whole brain in the same inter-commissural plane with the following parameters: $\mathrm{TR}=3000 \mathrm{~ms}, \mathrm{TE}=40 \mathrm{~ms}$, slice thickness $=3 \mathrm{~mm}$, slice skip $0.3 \mathrm{~mm}, \mathrm{NEX}=8$, image acquisition matrix $128 \times 128$, yielding an inplane spatial resolution of $1.875 \times 1.875 \mathrm{~mm}$. 


\section{Data analysis}

Age, years of education and clinical measures were compared between groups by means of Student's $t$ tests. The association between gender and group was calculated by means of a $\chi^{2}$ statistic in a $2 \times 2$ contingency table. The subjective fatigue and anxiety scores were analysed by means of separate ANOVAs including a between-participants factor - Group (control, CFS), and a within-participants factor - Situation (Relax, Fatigue, Anxiety).

\section{fMRI data analysis}

The fMRI data were analysed with software developed at the Institute of Psychiatry (XBAM), using a non-parametric approach to minimize assumptions. Data were first corrected for subject motion (Bullmore et al. 1999a) and then smoothed using a Gaussian filter [full width at half maximum (FWHM) $7.2 \mathrm{~mm}$ ] chosen to improve signal-to-noise ratio over the spatial neighbourhood of each voxel. Responses to the experimental paradigms were then detected by timeseries analysis using a linear model in which each component of the experimental design was convolved separately with two gamma variate functions (peak responses at $4 \mathrm{~s}$ and $8 \mathrm{~s}$ respectively) to permit variability in the haemodynamic delay. The method of Friman et al. (2003) was used to constrain model fits to those deemed physiologically plausible. Following computation of the model fit, a goodness-of-fit statistic was computed. This consisted of the ratio of the sum of squares of deviations from the mean image intensity due to the model (over the whole timeseries) to the sum of squares of deviations due to the residuals (SSQratio). This addresses the problem inherent in the use of the $F$ statistic that the residual degrees of freedom are often unknown in fMRI timeseries because of the presence of coloured noise in the signal. Following computation of the observed SSQratio at each voxel, the data were permuted by the wavelet-based method described and characterized extensively in Bullmore et al. (2001), which permits the data-driven calculation of the null distribution of SSQratios under the assumption of no experimentally determined response. This distribution can then be used to threshold the activation maps at any desired type I error rate. In addition to the SSQratio, the percentage blood oxygen level dependent (BOLD) change was also calculated from the model fit at each voxel.

The detection of activated regions was extended from voxel to cluster level using the method described in detail by Bullmore et al. (2001). The observed and randomized SSQratio data for each individual were transformed into standard space (see Talairach \&
Tournoux, 1988) and group maps of activated regions were computed using the median observed and randomized SSQratio data as described by Brammer et al. (1997). Permutation methods and median statistics were used to allow exact computation of $p$ values with minimal assumptions and the minimization of outlier effects. The hierarchical method of analysis used above also allows separate treatment of intraand inter-individual variance. After extension of inference from voxel to cluster level (Bullmore et al. $1999 b)$, the resulting cluster maps were thresholded to give $<1$ expected type I error cluster per whole brain volume to make interpretation of maps as intuitive as possible.

\section{Between-group comparisons}

For each level of the task, comparison of responses between groups was performed by fitting the data at each intra-cerebral voxel at which all subjects have non-zero data using a linear model of the type $Y=a+b X+e$, where $Y$ is the vector of BOLD effect sizes for each individual, $X$ is the contrast matrix for the particular intergroup contrasts required, $a$ is the mean effect across all individuals in the various groups, $b$ is the computed group difference and $e$ is a vector of residual errors. The model is fitted by minimizing the sum of absolute deviations rather than the sums of squares to reduce outlier effects. The null distribution of $b$ is computed by permuting data between groups (assuming the null hypothesis of no effect of group membership) and refitting the above model 50 times at each voxel and combining the data over all intra-cerebral voxels. Group difference maps are at any desired voxel or the cluster-wise type I error rate can then be computed by appropriate thresholding of this null distribution.

\section{Results \\ Demographic and clinical measures}

CFS patients and controls did not differ in terms of age, sex distribution or years of education. However, the patients' scores on all the clinical measures were within the severe range and were significantly higher than those of the controls (Table 1).

\section{Success of the fatigue provocation task}

Regarding subjective fatigue scores, both patients and controls rated the fatigue-provoking situations as more fatiguing than anxiety-provoking situations, and both were rated as more fatiguing than the relaxing situations [Situation $F(2,42)=56.14, p<0.001$ ]. In all 
Table 1. Comparison between CFS patients and controls on demographic and clinical variables

\begin{tabular}{llll}
\hline & $\begin{array}{l}\text { CFS patients } \\
(n=12) \\
\text { Mean (s.D.) }\end{array}$ & $\begin{array}{l}\text { Controls } \\
(n=11) \\
\text { Mean (s.D.) }\end{array}$ & \\
\hline \% Women & 66.7 & 63.6 & $\chi^{2}=0.02, \mathrm{df}=1, p=0.879$ \\
Age (years) & $33.75(7.64)$ & $34.36(6.77)$ & $t(21)=0.203, p=0.841$ \\
Years of education & $15.50(3.42)$ & $16.81(1.53)$ & $t(15.5)^{\mathrm{a}}=1.207, p=0.245$ \\
ChFS & $10.00(2.00)$ & $0.81(1.07)$ & $t(21)=13.51, p<0.001$ \\
WSAS & $4.74(1.64)$ & $0(0)$ & - \\
d-HADS & $8.16(2.88)$ & $1.81(2.27)$ & $t(21)=5.82, p<0.001$ \\
a-HADS & $10.66(2.74)$ & $4.72(2.53)$ & $t(21)=5.38, p<0.001$ \\
PF-SF36 & $48.75(19.90)$ & $95.00(5.00)$ & $t(12.5)^{\mathrm{a}}=7.78, p<0.001$ \\
\hline
\end{tabular}

CFS, Chronic fatigue syndrome; S.D., standard deviation; df, degrees of freedom; ChFS, Chalder Fatigue Scale; WSAS, Work and Social Adjustment Scale; d-HADS, depression scale from the Hospital Anxiety and Depression Scale; a-HADS, anxiety scale from the Hospital Anxiety and Depression Scale; PF-SF36, Physical Functioning scale from the 36-item Short Form Health Survey.

${ }^{a}$ Degrees of freedom were corrected after Levene's test.

b Statistics were not computed because all the controls scored 0 on this scale.

Table 2. Mean and standard deviations of the fatigue and anxiety scores reported by chronic fatigue syndrome (CFS) patients and controls after each type of imagined situation

\begin{tabular}{llll}
\hline & \multicolumn{3}{l}{ Imagined situation } \\
\cline { 2 - 4 } & Fatiguing & $\begin{array}{l}\text { Anxiety } \\
\text { provoking }\end{array}$ & Relaxing \\
\hline & & & \\
\hline Fatigue scores & $8.33(0.74)$ & $5.54(2.46)$ & $3.48(1.44)$ \\
$\quad$ CFS & $5.93(1.14)$ & $2.54(2.60)$ & $1.44(1.40)$ \\
$\quad$ Controls & & & \\
Anxiety scores & $5.22(1.84)$ & $8.60(1.50)$ & $1.36(0.65)$ \\
$\quad$ CFS & $2.88(1.71)$ & $8.25(1.05)$ & $0.88(0.81)$ \\
Controls & & & \\
\hline
\end{tabular}

three situations, patients reported higher levels of fatigue than controls [Group $F(1,21)=22.26, p<0.001$ ]. The Situation $\times$ Group interaction was not significant $[F(2,42)=0.579, p>0.1]$ (Table 2).

Analysis of the subjective anxiety scores revealed a slightly different picture. Again, both main effects Group $[F(1,21)=19.26, \quad p<0.05]$ and Situation $[F(2,42)=230.99, p<0.001]$ were significant. However, they were subjected to a significant Situation $\times$ Group interaction $[F(2,42)=5.31, p<0.01]$. Pairwise comparisons revealed no differences in levels of anxiety between patients and controls when presented with relaxing- and anxiety-provoking situations $(p>0.1)$, but patients experienced higher levels of anxiety during the fatiguing situations $[t(21)=3.15, p<0.005]$. Both patients and controls reported being more anxious during the anxiety-provoking situations than the fatiguing situations, both being more anxiety provoking than the relaxing situations [the smaller test was $t(10)=4.89, p<0.005$ ] (Table 2).

\section{fMRI results}

The BOLD responses associated with the fatiguing and anxiety-provoking situations were calculated using the relaxing videos as the contrast condition.

\section{Fatiguing videos}

Taking the 23 participants together, the fatigue $>$ relaxation contrast produced a large significant cluster with its peak in the fusiform gyrus, which included most of the occipito-parietal cortex, extending towards the cerebellum (1183 voxels; peak at $x=47, y=-63$, $\mathrm{Z}=-13 ; p=0.0009$ ) (see Table 3 for a breakdown of this cluster).

When comparing BOLD responses during the fatiguing situations between CFS patients and controls, the former showed greater activation than controls in a cluster with its peak in the precuneus [Brodmann area (BA) 7; 425 voxels; peak at $x=0$, $\mathrm{y}=-67, \mathrm{z}=53 ; p=0.00003$ ] but extending towards the bilateral posterior cingulate cortex (BA 23/29/31), left thalamus, the left fusiform (BA 19) and lingual gyri (BA 18); a second cluster in the right precuneus and cuneus (BA $7 ; 32$ voxels; peak at $x=4, y=-59$, 
Table 3. Brain areas with significant blood oxygen level dependent $(B O L D)$ response resulting from the contrasts Fatigue $>$ Relaxation and Anxiety $>$ Relaxation, including the whole sample

\begin{tabular}{|c|c|c|c|c|c|}
\hline & BA & $\mathrm{x}$ & $\mathrm{y}$ & $\mathrm{z}$ & Size \\
\hline \multicolumn{6}{|c|}{ Fatigue provocation } \\
\hline \multicolumn{6}{|c|}{ Cerebellum } \\
\hline $\mathrm{R}$ & - & 11 & -67 & -29 & 101 \\
\hline $\mathrm{L}$ & - & -25 & -70 & -35 & 20 \\
\hline \multicolumn{6}{|c|}{ Fusiform gyrus } \\
\hline $\mathrm{R}$ & 18 & 40 & -70 & -13 & 113 \\
\hline $\mathrm{L}$ & 37 & -47 & -63 & -18 & 22 \\
\hline \multicolumn{6}{|c|}{ Lingual gyrus } \\
\hline $\mathrm{R}$ & 18 & 18 & -81 & -7 & 126 \\
\hline $\mathrm{L}$ & 18 & -22 & -78 & 4 & 13 \\
\hline \multicolumn{6}{|c|}{ Precuneus } \\
\hline $\mathrm{R}$ & 7 & 4 & -70 & 37 & 37 \\
\hline \multicolumn{6}{|c|}{ Cuneus } \\
\hline $\mathrm{R}$ & $18 / 19$ & 22 & -74 & 20 & 58 \\
\hline \multicolumn{6}{|c|}{ Paracentral lobule } \\
\hline $\mathrm{L}$ & 7 & -4 & -48 & 64 & 16 \\
\hline \multicolumn{6}{|c|}{ Anxiety provocation } \\
\hline \multicolumn{6}{|c|}{ Cerebellum } \\
\hline $\mathrm{R}$ & - & 40 & -59 & -29 & 11 \\
\hline $\mathrm{L}$ & - & -47 & -59 & -24 & 19 \\
\hline \multicolumn{6}{|c|}{ Fusiform gyrus } \\
\hline $\mathrm{R}$ & 37 & 43 & -56 & -13 & 38 \\
\hline $\mathrm{L}$ & 37 & -51 & -59 & -13 & 29 \\
\hline \multicolumn{6}{|c|}{ Lingual gyrus } \\
\hline $\mathrm{L}$ & 18 & -22 & -85 & -2 & 16 \\
\hline \multicolumn{6}{|c|}{ Inferior temporal gyrus } \\
\hline $\mathrm{R}$ & 37 & 43 & -52 & -7 & 42 \\
\hline $\mathrm{L}$ & 37 & -51 & -59 & -7 & 34 \\
\hline \multicolumn{6}{|c|}{ Medial temporal gyrus } \\
\hline $\mathrm{R}$ & 21 & 51 & -44 & 4 & 12 \\
\hline $\mathrm{L}$ & 37 & -51 & -59 & -2 & 16 \\
\hline \multicolumn{6}{|c|}{ Superior temporal gyrus } \\
\hline $\mathrm{R}$ & 22 & 58 & -30 & 9 & 12 \\
\hline $\mathrm{L}$ & 22 & -58 & -37 & 9 & 14 \\
\hline \multicolumn{6}{|c|}{ Posterior cingulate gyrus } \\
\hline $\mathrm{L}$ & 23 & -4 & -48 & 26 & 4 \\
\hline \multicolumn{6}{|c|}{ Precuneus } \\
\hline B & 7 & 0 & -63 & 48 & 18 \\
\hline \multicolumn{6}{|c|}{ Cuneus } \\
\hline B & $18 / 19$ & 0 & -78 & 15 & 23 \\
\hline
\end{tabular}

BA, Brodmann area; R, right; B, bilateral; L, left.

Only the clusters with the largest number of voxels within each region are reported. The Talairach coordinates correspond to the most activated voxel in each cluster.

$\mathrm{z}=59 ; p=0.0008)$; and a third cluster in the left cerebellum (7 voxels; peak at $\mathrm{x}=-25, \mathrm{y}=-74$, $\mathrm{z}=-35 ; p=0.0049)$ (Fig. 2).
The healthy controls showed greater activation than the CFS patients in a cluster with its peak at the right superior dorsolateral prefrontal cortex (BA $8 ; 186$ voxels; peak at $\mathrm{x}=29, \mathrm{y}=22, \mathrm{z}=48 ; p=0.0002)$, extending towards the right middle frontal gyrus (BA 9), the medial parts of the frontal gyrus (BA 6), the right insula/superior temporal gyrus (BA 22/42), and the right precentral gyrus (BA 4) (Fig. 2).

\section{Anxiety-provoking videos}

In the patients and controls combined, the anxiety $>$ relaxation contrast revealed a significant cluster with its peak in the left parietal cortex (BA 7; 297 voxels, peak at $\mathrm{x}=-4, \mathrm{y}=-52, \mathrm{z}=64, p=0.005)$; a second cluster with its peak at the fusiform gyrus (BA 37 )/cerebellum (220 voxels, peak at $x=-51, y=-59$, $\mathrm{z}=-18 ; p=0.005)$; and a similar right-sided cluster with its peak at the right inferior temporal gyrus (BA 37 ; 217 voxels, peak at $\mathrm{x}=43, \mathrm{y}=-52, \mathrm{z}=-7$; $p=0.005$ ) (see Table 3 for a breakdown of these clusters).

CFS patients showed greater activation than controls in a cluster (227 voxels; peak at $x=-18, y=44$, $\mathrm{z}=-13 ; p=0.0002)$ occupying the bilateral ventral prefrontal cortex (BA 10/11/47) and medial prefrontal cortex including the anterior cingulate (BA 9/10/24/ 32) (Fig. 3). However, controls showed greater activation than CFS patients in a cluster (67 voxels; peak at $\mathrm{x}=-51, \mathrm{y}=-44, \mathrm{z}=20 ; p=0.001)$ occupying the left superior temporal gyri (BA 22/41/42) and extending towards the inferior parietal/postcentral gyrus (BA 40) and precentral gyrus (BA 4).

Within-group analyses (differences between video contents)

In the CFS group $(n=12)$, the fatigue-provoking videos resulted in significantly greater BOLD responses than the anxiety-provoking videos in the cerebellum and occipito-parietal regions bilaterally (BA 7, 17, 18, 19) extending towards the dorsal and posterior parts of the cingulate gyrus bilaterally (BA 24, 29, 31), the left hippocampal gyrus (BA 36) and the left caudate nucleus (Fig. 4). The opposite contrast yielded activation in the bilateral anterior cingulate cortex (BA 24, 32) and medial parts of the frontal cortex (BA 6, 10), in the inferior frontal gyrus [BA 44, 45 (right) and 47 (left)] and superior temporal gyrus (BA 38) extending towards the bilateral insula, and towards the right precentral gyrus (BA 6) and basal ganglia (Fig. 4).

In the healthy control group, the fatigue-provoking videos produced greater BOLD responses than the anxiety-provoking videos in the right dorsolateral (BA $9,46)$ and bilateral medial (BA 6, 10) prefrontal cortex extending towards the anterior cingulate (BA 24, 32), right insula and right caudate nucleus (Fig. 4). In 

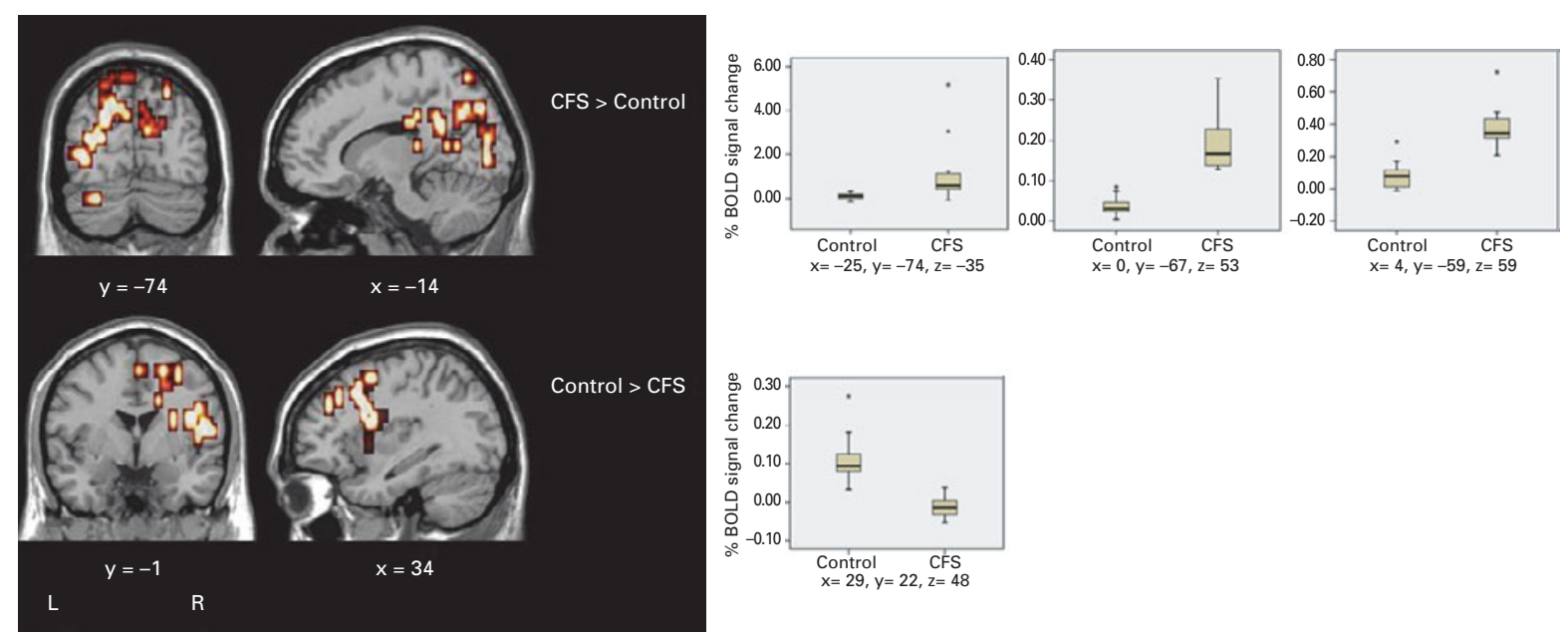

Fig. 2. Differences in activation between chronic fatigue syndrome (CFS) patients and controls during the fatigue-inducing scenarios. CFS patients showed greater activation than controls in the cerebellum (peak at $\mathrm{x}=-25, \mathrm{y}=-74, \mathrm{z}=-35 ; 7$ voxels; $p=0.0049$ ), bilateral parietal cortex [peak at $\mathrm{x}=0, \mathrm{y}=-67, \mathrm{z}=53$; Brodmann area (BA) $7 ; 425$ voxels; $p=0.00003$ ] and right precuneus (peak at $\mathrm{x}=4, \mathrm{y}=-59, \mathrm{z}=59 ; \mathrm{BA} 7 ; 32$ voxels; $p=0.0008$ ). Controls showed greater activation in the right dorsolateral prefrontal cortex (peak at $\mathrm{x}=29, \mathrm{y}=22, \mathrm{z}=48$; BA $8 ; 186$ voxels; $p=0.0002$ ). Each box plot shows the median (horizontal line), quartiles (box) and extreme values (whiskers). This figure is for display purposes only and indicates the most representative slices. $L$, left; $R$, right.


Fig. 3. Differences in activation between chronic fatigue syndrome (CFS) patients and controls during the anxiety-inducing scenarios. CFS patients showed greater activation than controls in a cluster with its peak in the left ventral prefrontal cortex [peak at $\mathrm{x}=-18, \mathrm{y}=44, \mathrm{z}=-13$; Brodmann area (BA) 11, 227 voxels; $p=0.0002$ ]. Controls showed greater activation than CFS patients in a cluster with its peak in the superior temporal lobe/lateral sulcus (peak at $\mathrm{x}=-51, \mathrm{y}=-44, \mathrm{z}=20$; $\mathrm{BA}$ $22 / 42 ; 67$ voxels; $p=0.001$ ). Each box plot shows the median (horizontal line), quartiles (box) and extreme values (whiskers). This figure is for display purposes only and indicates the most representative slices. L, left; R, right.

addition, the cerebellum and right occipital cortex (BA 18,19 ) including the fusiform gyrus (BA 37) bilaterally were significantly more activated in the fatigue- than the anxiety-provoking videos. The anxiety-provoking videos produced greater activity than the fatiguing ones in the left temporal lobe (BA 20,37,39), extending towards parietal (BA 7,19) and visual areas (BA 18) (Fig. 4).

\section{Discussion}

To our knowledge, this is the first study to examine the neural correlates of experimentally induced feelings of fatigue in CFS and healthy controls. We used a novel symptom provocation procedure consisting of the presentation of audio instructions and fatigueinducing videos designed to mimic real-life situations. 




Fig. 4. Within-group comparisons of the activations obtained in the fatigue- and anxiety-inducing scenarios. In the chronic fatigue syndrome (CFS) group, the fatigue $>$ anxiety contrast produced greater activation in the cerebellum and occipito-parietal regions extending towards the posterior cingulate gyrus. The anxiety $>$ fatigue contrast yielded greater activation in the anterior cingulate cortex and medial parts of the frontal cortex extending towards the insula, the right precentral gyrus and the basal ganglia. In the control group, the fatigue $>$ anxiety contrast produced greater activations in the dorsolateral and medial prefrontal cortex, anterior cingulate gyrus, insula and caudate nucleus. The anxiety $>$ fatigue contrast produced greater activity in the temporal and parietal lobes and visual areas. This figure is for display purposes only and indicates the most representative slices. $\mathrm{L}$, left; $\mathrm{R}$, right.

We also included a non-fatiguing anxiety-provoking condition in the experiment to control for the nonspecific effects of anxiety.

Pilot work and analysis of the current data confirmed that the symptom provocation procedure was effective. Fatigue-inducing videos yielded higher subjective fatigue scores than anxiety-inducing videos and, conversely, anxiety-inducing videos yielded higher subjective anxiety scores than fatigue-inducing videos. CFS patients found all types of videos more fatigue inducing than healthy controls. Patients and controls did not differ in their subjective ratings of anxiety during the anxiety-provoking scenarios, but, interestingly, CFS patients experienced higher rates of anxiety during the fatiguing scenarios, suggesting that feelings of fatigue and anxiety are intimately linked in CFS.

Turning to the fMRI results, in patients and controls combined, the provocation of imaginal fatigue resulted in large widespread activations in posterior brain regions, mainly occipital and parietal cortices. Between-group analyses revealed that CFS patients showed significantly greater activation in these brain regions than healthy controls. In addition, CFS patients also showed greater activations in the posterior cingulate cortex. Within-group analyses showed that CFS patients activated these same regions significantly more during the fatiguing than the anxietyprovoking videos. Similar brain regions correlated with subjective fatigue in a recent study that used a cognitively challenging task (Cook et al. 2007). These brain regions have consistently been implicated in the processing of emotional stimuli and the retrieval of emotional memories (Nielsen et al. 2005; Garret \& Maddock, 2006; Pourtois et al. 2006; Smith et al. 2006) using a variety of experimental paradigms. The results seem to suggest that the provocation of fatigue in patients with CFS is associated with activation in brain regions implicated in emotion processing and the retrieval of emotional memories. This is consistent with the cognitive-behavioural model of CSF, which proposes that fear and avoidance are key maintaining factors for the disorder (Chalder et al. 2000). It could be argued that patients remember feeling severely fatigued in the past and that they are anxious about feeling so fatigued again. It is the fear of feeling fatigue that can lead them to avoid activity.

During the provocation of fatigue, the CFS group also showed reduced activations in dorsolateral and dorsomedial prefrontal regions (BA 6/8/9), compared with the healthy controls. These prefrontal cortical regions form part of a network that has been associated 
with inhibitory processes and emotion regulation (Ochsner et al. 2002; Phillips et al. 2003; Phan et al. 2005). Our results suggest that CFS patients may experience difficulties down-regulating or suppressing the emotional states associated with feelings of fatigue.

These results do not support our initial hypothesis that CFS patients would show exaggerated neural responses in brain areas previously associated with pain perception and conscious interoception (i.e. anterior cingulate, insula and somatosensory cortex). In fact, the reverse was true; controls showed greater activations than CFS patients in these regions. The reasons for this are unclear but it could be speculated that during the imaginal fatigue condition, the patients' attentional resources are primarily allocated to the highly salient stimuli at the expense of a conscious processing of interoceptive information (Critchley, 2004). It is also plausible that this may lead to CFS patients misinterpreting anxiety or stress as fatigue or the failure to recognize that the fatigue is a symptom of the underlying anxiety/stress response. This misinterpretation could be related to difficulties in identifying and/or accepting emotions (Creswell \& Chalder, 2001).

Analysis of the anxiety-provoking condition revealed that CFS patients showed increased activations in both ventral (BA 10/11/47) and dorsal (BA 9/10/ 24/32) prefrontal regions compared with healthy controls. The ventral prefrontal regions have been implicated in numerous studies using a variety of emotional paradigms (see Phan et al. 2002 for a metaanalysis), whereas the dorsal prefrontal regions have been implicated in emotional regulation (Ochsner et al. 2002; Phillips et al. 2003; Phan et al. 2005). Withingroup analyses revealed that these regions were significantly more activated in the anxiety- than the fatigue-provoking condition among CFS patients and that the opposite was true in the control group. These results seem to suggest more general emotionprocessing alterations in CFS that are not exclusively associated with the provocation of fatigue. This would be consistent with recent reports that have demonstrated that early adverse experience, stress and emotional instability are important risk factors for the development of the disorder (Heim et al. 2006; Kato et al. 2006). It is possible that although CFS patients fail to down-regulate their emotional responses to fatiguing situations (as discussed above), they may tend to over-regulate their emotional responses to more general emotional stimuli. Indeed, a recent study has shown that engaging in emotional processing during therapy was significantly associated with a good therapeutic outcome in CFS (Godfrey et al. 2007). This hypothesis would be consistent with a previous study that showed that patients with CFS tend to suppress their emotional responses in general (Creswell \& Chalder, 2001).

The main limitation of the current investigation was its relatively small sample. However, the patients were well characterized, unmedicated, and free of any co-morbid psychiatric disorder. Although we used robust non-parametric methods of statistical analysis that minimize the probability of false-positive results, the results require replication in a larger patient sample. The diagnostic specificity of the findings remains to be established by comparing CFS with other emotional disorders. It is possible that CFS patients could find the whole scanning process more fatiguing than controls. This is a common problem in many neuroimaging studies of emotional disorders. For example, the scanning process is likely to be more anxiety provoking for anxious patients than for controls. Future research using absolute rather than relative measures of brain activity is warranted to control for this potential problem. Finally, the temporal stability of the findings will require testing using repeat administrations of this paradigm before and after treatment.

\section{Acknowledgements}

This research was funded by grants from the South London and Maudsley NHS Trust (Drs Godfrey and Mataix-Cols), the Spanish Ministry of Education and Science (Dr X. Caseras, grant no. EX-2004-1137), and the Get Well Club, Surrey. Professor Peter White and Giselle Whiters helped with the recruitment of one participant at St Bartholomew's Hospital.

\section{Declaration of Interest}

None.

\section{References}

Angst J, Dobler-Mikola A (1985). The Zurich Study. V. Anxiety and phobia in young adults. European Archives of Psychiatry and Neurological Sciences 235, 171-178.

Beauregard M, Paquette V, Levesque J (2006). Dysfunction in the neural circuitry of emotional self-regulation in major depressive disorder. Neuroreport 17, 843-846.

Brammer M, Bullmore ET, Simmons A, Williams SCR, Grasby PM, Howard RJ, Woodruff PWR, Rabe-Hesketh S (1997). Generic brain activation mapping in functional magnetic resonance imaging: a nonparametric approach. Magnetic Resonance Imaging 15, 763-770.

Bullmore ET, Brammer M, Rabe-Hesketh S, Curtis V, Morris R, Williams SCR, Sharma T, McGuire PK (1999a). Methods for the diagnosis and treatment of stimulus 
correlated motion in generic brain activation studies using fMRI. Human Brain Mapping 7, 38-48.

Bullmore ET, Long C, Suckling J, Fadili J, Calvert GA, Zelaya F, Carpenter TA, Brammer MJ (2001). Coloured noise and computational inference in neurophysiological (fMRI) time series analysis: resampling methods in time and wavelet domains. Human Brain Mapping 12, 61-78.

Bullmore ET, Suckling J, Overmeyer S, Rabe-Hesketh S, Taylor E, Brammer MJ (1999b). Global, voxel and cluster tests, by theory and permutation, for a difference between two groups of structural MR images of the brain. IEEE Transactions on Medical Imaging $18,32-42$.

Caseras X, Mataix-Cols D, Giampietro V, Rimes KA, Brammer M, Zelaya F, Chalder T, Godfrey EL (2006). Probing the working memory system in chronic fatigue syndrome: a functional magnetic resonance imaging study using the n-back task. Psychosomatic Medicine 68, 947-955.

Chalder T, Berelowitz G, Pawlikowska T, Watts L, Wessely S, Wright D, Walalce EP (1993). Development of a fatigue scale. Journal of Psychosomatic Research 37, 147-153.

Chalder T, Cleare A, Wessely S (2000). The management of stress and anxiety in chronic fatigue syndrome. In The Management of Stress and Anxiety in Medical Disorders (ed. D. I. Mostofsky and D. H. Barlow), pp. 160-179. Alleyn and Bacon: Boston.

Cook DA, O'Connor PJ, Lange G, Steffener J (2007). Functional neuroimaging correlates of mental fatigue induced by cognition among chronic fatigue syndrome patients and controls. NeuroImage 36, 108-122.

Creswell C, Chalder T (2001). Defensive coping styles in chronic fatigue syndrome. Journal of Psychosomatic Research 51, 607-610.

Critchley HD (2004). The human cortex responds to an interoceptive challenge. Proceedings of the National Academy of Sciences USA 101, 6333-6334.

Deale A, Chalder T, Wessely S (1998). Illness beliefs and outcome in chronic fatigue syndrome: is change in causal attribution necessary for clinical improvement? Journal of Psychosomatic Research 45, 77-83.

Fischler B, Cluydts R, De Gucht V, Kaufman L, DeMeirleir K (1997). Generalised anxiety disorder in chronic fatigue syndrome. Acta Psychiatrica Scandinavica 95, 405-413.

Friman O, Borga M, Lundberg P, Knutsson H (2003). Adaptive analysis of fMRI data. NeuroImage 19, 837-845.

Fukuda K, Straus SE, Hickie I, Sharpe MC, Dobbins JG, Komaroff A (1994). The chronic fatigue syndrome: a comprehensive approach to its definition and study. International Chronic Fatigue Syndrome Study Group. Annals of Internal Medicine 121, 953-959.

Garret AS, Maddock RJ (2006). Separating subjective emotion from the perception of emotion-inducing stimuli: an fMRI study. NeuroImage 33, 263-274.

Georgiades E, Behan WMH, Kilduff LP, Hadjicharalambous M, Mackie EE, Wilson J, Ward SA, Pitsiladis YP (2003). Chronic fatigue syndrome: new evidence for a central fatigue disorder. Clinical Science $\mathbf{1 0 5}$ 213-218.

Godfrey E, Chalder T, Ridsdale L, Seed P, Ogden J (2007). Investigating the 'active ingredients' of cognitive behaviour therapy and counselling for patients with chronic fatigue in primary care; developing a new process measure to assess treatment fidelity and predict outcome. British Journal of Clinical Psychology 46, 253-272.

Heim C, Wagner D, Maloney E, Papanicolaou DA, Solomon L, Jones JF, Unger ER, Reeves WC (2006). Early adverse experience and risk for chronic fatigue syndrome: results from a population-based study. Archives of General Psychiatry 63, 1258-1266.

Kato K, Sullivan PF, Evengard B, Pedersen NL (2006). Premorbid predictors of chronic fatigue. Archives of General Psychiatry 63, 1267-1272.

Kent-Braun JA, Sharma KR, Weiner MW, Massie B, Miller RG (1993). Central basis of muscle fatigue in chronic fatigue syndrome. Neurology 43, 125-131.

Lange G, Steffener J, Cook DB, Bly BM, Christodoulou C, Liu WC, Deluca J, Natelson BH (2005). Objective evidence of cognitive complaints in chronic fatigue syndrome: a BOLD fMRI study of verbal working memory. NeuroImage 26, 513-524.

Mataix-Cols D, Cowley AJ, Hankins M, Schneider A, Bachofen M, Kenwright M, Gega L, Cameron R, Marks IM (2005). Reliability and validity of the Work and Social Adjustment Scale in phobic disorders. Comprehensive Psychiatry 46, 223-228.

Mataix-Cols D, Wooderson S, Lawrence N, Brammer MJ, Speckens A, Phillips ML (2004). Distinct neural correlates of washing, checking, and hoarding symptom dimensions in obsessive-compulsive disorder. Archives of General Psychiatry 6, 564-576.

Mundt JC, Marks IM, Greist JH, Shear K (2002). Work and Social Adjustment Scale: a simple accurate measure of impairment in functioning. British Journal of Psychiatry 180, 461-464.

Nielsen FA, Balslev D, Hansen LK (2005). Mining the posterior cingulate: segregation between memory and pain components. NeuroImage 27, 520-532.

Ochsner KN, Bunge SA, Gross JJ, Gabrieli JD (2002). Rethinking feelings: an fMRI study of the cognitive regulation of emotion. Journal of Cognitive Neuroscience 14, 1215-1229.

Phan KL, Fitzgerald DA, Nathan PJ, Moore GJ, Uhde TW, Tancer ME (2005). Neural substrates for voluntary suppression of negative affect: a functional magnetic resonance imaging study. Biological Psychiatry 57, 210-219.

Phan KL, Wager T, Taylor SF, Liberzon I (2002). Functional neuroanatomy of emotion: a meta-analysis of emotion activation studies in PET and fMRI. NeuroImage 16, 331-348.

Phillips ML, Drevets WC, Rauch SL, Lane R (2003). Neurobiology of emotion perception. I: Implications for major psychiatric disorders. Biological Psychiatry 54, 515-528.

Pourtois G, Schwartz S, Seghier ML, Lazeyras F, Vuilleumier P (2006). Neural systems for orienting 
attention to the location of threat signals: an event-related fMRI study. NeuroImage 31, 920-933.

Sheehan DV, Lecrubier Y, Sheehan KH, Amorim P, Janavs J, Weiller E, Hergueta T, Barker R, Dunbar GC (1998).

The Mini-International Neuropsychiatric Interview (M.I.N.I.): the development and validation of a structured diagnostic psychiatric interview for DSM-IV and ICD-10. Journal of Clinical Psychiatry 59 (Suppl. 20), 22-33.

Silver A, Haeney M, Vijayadurai P, Wilks D, Pattrick M, Main CJ (2002). The role of fear of physical movement and activity in chronic fatigue syndrome. Journal of Psychosomatic Research 52, 485-493.

Smith AP, Stephan KE, Rugg MD, Dolan RJ (2006). Task and content modulate amygdale-hippocampal connectivity in emotional retrieval. Neuron 49, 631-638.

St Clair Gibson A, Baden DA, Lambert MI, Lambert EV, Harley YX, Hampson D, Russell VA, Noakes TD (2003). The conscious perception of the sensation of fatigue. Sports Medicine 33, 167-176.
Talairach J, Tournoux P (1988). Co-planar Stereotactic Atlas of the Human Brain. Thieme: Stuttgart.

van der Werf SP, Prins JB, Vercoulen JHMM, van der Meer JWM, Bleijenberg G (2000). Identifying physical activity patterns in chronic fatigue syndrome using actigraphic assessment. Journal of Psychosomatic Research 49, 373-379.

Ware JE, Sherbourne C (1992). The MOS 36-item short form health survey (SF-36). Medical Care 30, 473-483.

Whiteside A, Hansen S, Chaudhuri A (2004). Exercise lowers pain threshold in chronic fatigue syndrome. Pain 109, 497-499.

Whiting P, Bagna AM, Sowden AJ, Cornell JE, Mulrow CD, Ramírez G (2001). Interventions for the treatment and management of chronic fatigue syndrome. Journal of the American Medical Association 286, 1360-1368.

Zigmond AS, Snaith RP (1983). The Hospital Anxiety and Depression Scale. Acta Psychiatrica Scandinavica 67, 361-370. 